\title{
Spontaneous Massive Adrenal Hemorrhage: A Management Dilemma
}

\author{
Praveen Pushkar, MS and Anshuman Agarwal, MCh
}

\begin{abstract}
Adrenal hemorrhage (AH) is a rare but life-threatening condition. Small focal hemorrhage may present subclinically, but massive hemorrhage may lead to rapid cardiovascular collapse and ultimately death if not diagnosed appropriately and treated quickly. Most cases reported in the literature have been treated conservatively. In an event of increasing hemorrhage during conservative management, it may be tricky to intervene surgically because of the hematoma around the gland. Here we describe a case where we managed a large spontaneous $\mathrm{AH}$ by a combination of angioembolization and laparoscopic adrenalectomy.
\end{abstract}

\section{Introduction and Background}

A DRENAL HEMORRHAGE (AH) is a rare but lifethreatening condition, especially when it occurs bilaterally. Etiology includes both traumatic and nontraumatic events. Clinical manifestation depends on the degree and rate of hemorrhage. Small focal hemorrhage may present subclinically, but massive hemorrhage may lead to rapid cardiovascular collapse and ultimately death if not diagnosed appropriately and treated quickly. Most cases reported in the literature have been treated conservatively. In an event of increasing hemorrhage during conservative management, it may be tricky to intervene surgically because of the hematoma around the gland. Here we describe a case where we managed a large spontaneous AH by a combination of angioembolization and laparoscopic adrenalectomy. To the best of our knowledge, it has not been reported in the literature yet.

\section{Presentation of Case}

A 26-year-old, nonhypertensive young lad presented to us in emergency with left-sided acute abdominal pain of 1 day duration. Pain was sudden in onset, severe, continuous, and nonradiating. On initial examination, he was hemodynamically stable with blood pressure of $140 / 70 \mathrm{~mm} \mathrm{Hg}$ and heart rate of 110 beats per minute. On abdominal examination, he had a tender vague lump in the left upper quadrant of abdomen. Bowel sounds were normal. First, ultrasound (USG) abdomen and, later, contrast-enhanced CT scan abdomen were performed, which revealed hemorrhagic collection of $9 \times 8.2 \times 8.2 \mathrm{~cm}$ near the left suprarenal region with $4 \times 4.5 \times 4.2 \mathrm{~cm}$ adrenal mass. To evaluate the adrenal mass, MRI was performed, which showed the left suprarenal mass with large hemorrhagic component, suggestive of spontaneous hemorrhage in an adrenal mass (Fig. 1). Based on imaging finding, a working diagnosis of pheochromocytoma was made and he was started on phenoxybenzamine $10 \mathrm{mg}$ twice a day to prepare him for surgery. His

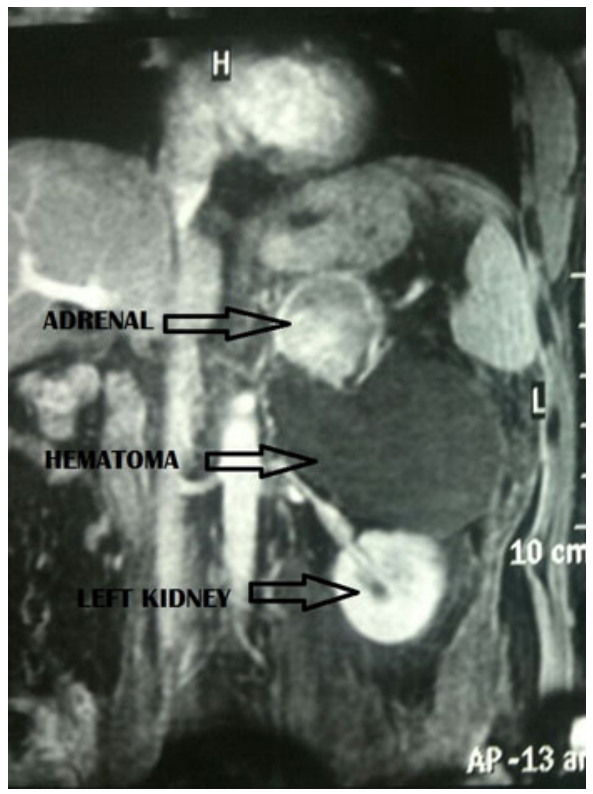

FIG. 1. MRI showing left suprarenal mass with large hemorrhagic component, suggestive of spontaneous hemorrhage in an adrenal mass, likely representing pheochromocytoma.

Department of Urology, Indraprastha Apollo Hospital, New Delhi, India.

(C) Pushkar and Agarwal 2015; Published by Mary Ann Liebert, Inc. This Open Access article is distributed under the terms of the Creative Commons License (http://creativecommons.org/licenses/by/4.0), which permits unrestricted use, distribution, and reproduction in any medium, provided the original work is properly credited. 


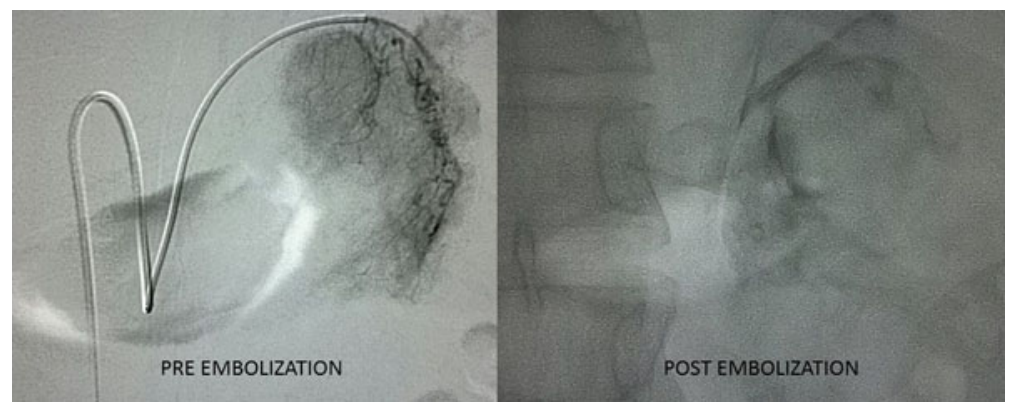

FIG. 2. Angiography revealed a highly vascular adrenal tumor. Two arteries were identified, one arising from aorta supplying superior part of gland and other arising from accessory left renal artery supplying the inferior part (preembolization). Both the arteries were embolized (postembolization).

urinary catecholamines were sent before starting phenoxybenzamine. He was kept in intensive care unit for close monitoring. There was one blood pressure recording of $>160 \mathrm{~mm} \mathrm{Hg}$ (systolic). He complained of resurgence in pain, so a bed-side USG of the abdomen was performed, which revealed an increase in collection by $500 \mathrm{~mL}$ resulting in a drop of $\mathrm{Hb}$ by $2 \mathrm{gm} / \mathrm{dL}$. He was transfused 2 units of packed red blood cells. Immediately, angioembolization was performed using Gelfoam (Fig. 2). No blood pressure variation was recorded during the procedure. Thirty-six hours later, he was taken up for laparoscopic exploration with adrenalectomy. Laparoscopy revealed a large collection of clotted blood in the retroperitoneum pushing the kidney inferiorly. Fortunately, there was no active hemorrhage. The ruptured adrenal gland was isolated after evacuation of the hematoma and removed after securing the pedicles. There was no blood pressure fluctuation during the surgery. Postoperative period remained uneventful. Results of urinary catecholamine levels came after the surgery and were normal. He was discharged on postoperative day 5. Histopathologic examination revealed adrenocortical adenoma based on the Weiss criteria. Follow-up performed after 6 and 12 months revealed no abnormality in positron emission tomography CT scan.

\section{Discussion and Literature Review}

AH may result from acute illness/stress, anticoagulation, coagulopathy, underlying tumor-like angiomyolipoma, trauma, or idiopathic disease. Most of the reported cases of $\mathrm{AH}$ in the literature are seen in pregnancy. These are usually intra-AHs. In young adults, idiopathic spontaneous $\mathrm{AH}$ is extremely rare and has never been reported. The presenting symptoms usually are hemorrhagic shock, flank pain, and fever.

$\mathrm{AH}$ has been reported in $0.3 \%$ to $1.8 \%$ of undetected cases in autopsy studies. ${ }^{1}$ Tumors known to cause spontaneous bleed are pheochromocytoma, myelolipoma, metastasis, carcinoma, and rarely adenoma. There are $\sim 50$ reported cases of spontaneous rupture of adrenal pheochromocytoma causing haemorrhage ${ }^{2}$ in the literature, but there is no reported case of adrenal adenoma causing massive bleeding.

Once adrenal pathology is suspected in a patient with retroperitoneal hemorrhage, he should be managed in an intensive care unit with close hemodynamic monitoring. Serial hematocrit and USG monitoring of size of hematoma has to be performed. Pheochromocytoma should always be kept in mind while dealing with such a case. MRI is the imaging modality of choice for diagnosis of nontraumatic $\mathrm{AH}$.

In patients with active bleeding, angiographic embolization is a valuable tool to achieve hemostasis. If complete hemostasis is achieved, patient is asymptomatic, and hemodynamically stable, then immediate surgical exploration should be avoided. But if patient deteriorates, surgical exploration may have to be performed. All preoperative principles performed in pheochromocytoma should be followed.

This is the first case report of such a massive spontaneous $\mathrm{AH}$ in adrenal adenoma in a young adult, which was effectively managed laparoscopically.

\section{Conclusion}

A high index of suspicion is required to make a timely diagnosis of $\mathrm{AH}$. Although acute surgical removal of an adrenal tumor within a large hematoma should be avoided, as proper oncologic resection may not be possible, it sometimes has to be performed in a symptomatic hemodynamically deteriorating patient.

\section{Disclosure Statement}

No competing financial interests exist.

\section{References}

1. Gavrilova-Jordan L, Edmister WB, Farrell MA, Watson WJ. Spontaneous adrenal hemorrhage during pregnancy: A review of literature and case report of successful conservative management. Obstet Gynecol Surv 2005;60:191-195.

2. Kobayashi T, Iwai A, Takahashi R, Ide Y, Nishizawa K, Mitsumori K. Spontaneous rupture of adrenal pheochromocytoma: Review and analysis of prognostic factors. J Surg Oncol 2005;90:31-35.

Address correspondence to: Praveen Pushkar, MBBS, MS Department of Urology Indraprastha Apollo Hospital

Sarita Vihar

New Delhi 110076

India

E-mail: praveenpushkar@yahoo.co.in

$$
\begin{aligned}
& \text { Abbreviations Used } \\
\mathrm{AH} & =\text { adrenal hemorrhage } \\
\mathrm{CT} & =\text { computed tomography } \\
\mathrm{MRI} & =\text { magnetic resonance imaging } \\
\mathrm{USG} & =\text { ultrasound }
\end{aligned}
$$

Cite this article as: Pushkar P, Agarwal A (2015) Spontaneous massive adrenal hemorrhage: a management dilemma, Journal of Endourology Case Reports 1:1, 52-53, DOI: 10.1089/cren.2015.0003. 